\title{
BAYESIAN APPROACH TO MODELLING OF QUASI-PERIODIC INTERMITTENT DEMAND
}

\author{
Alexandre Dolgui ${ }^{1}$, Anatoly Pashkevich ${ }^{1,2}$, Maxim Pashkevich ${ }^{3}$ \\ ${ }^{1}$ Division for Industrial Engineering and Computer Sciences (G2I) \\ Ecole de Mines de Saint Etienne, 158, Cours Fauriel, 42023 Saint Etienne, France \\ e-mail:dolgui@emse.fr \\ ${ }^{2}$ Robotic Laboratory, Department of Automatic Control \\ Belarusian State University of Informatics and Radioelectronics \\ 6 P.Brovka St., Minsk 220027, Belarus, e-mail: pap@bsuir.unibel.by \\ ${ }^{3}$ Department of Mathematical Modeling and Data Analysis \\ Belarusian State University, Skarina Av. 4, Minsk 220050, Belarus \\ e-mail:pma@omegasoftware.com
}

\begin{abstract}
The paper focuses on the stochastic modelling of the quasi-periodic intermittent demand patterns, which arise in the inventory management of the "slow moving items" such as service parts or high-priced capital goods. It is proposed a new stochastic model, which describes the demand patterns with essentially nonexponential distribution of the inter-arrival times. The model is based on generalized beta-binomial distribution and the Bayesian inference using the historical data array describing the demand repeatability within the time periods. For this model, there were derived explicit expressions for the forecast distributions, its moments and relevant Bayesian risk. The efficiency of the proposed approach is confirmed by computer simulation and an application example. Copyright $@ 2005$ IFAC
\end{abstract}

Keywords: inventory control, demand modelling, statistical inference, parameter estimation, prediction methods.

\section{INTRODUCTION}

Accurate demand forecasting is a principal component of supply chain management allowing companies to balance service levels against investment over large assortment of stock-keeping units (SKU) (Harris, 1997; Silver et al., 1998). As follows from previous research (Snyder, 2002), large portion of SKUs is usually made up by "slow moving items" with irregular (intermittent) demand patterns, i.e. random sequences with a large proportion of zeros and great variability among the remaining nonzero integer val- ues (Willemain et al., 2004). This paper addresses a special case of the intermittent demand possessing quasi-periodic regularity typical for many supply planning systems, from retail sales to manufacturing of high-priced capital goods.

The first heuristic technique for the intermittent demand forecasting was developed by Croston (1972) who applied separate exponential smoothing to both the non-zero demand sizes and inter-arrival times between successive demands. Later, the problem has been addressed by a number of authors (Johnston and 
Boylan, 1996, Syntetos and Boylan, 2001) who concentrated on improving the forecast accuracy and proposed several modifications of the Croston's method, including EWMA-smoothing and logtransformation of the data (both the demand values and inter-arrival times) in order to avoid the positivespace constraint. However, the corresponding model variables were still deterministic and defined on a continuous space. Consequently, all of these methods do not produce forecast distributions and associated prediction intervals (Shenstone and Hyndman, 2003).

The first stochastic demand models were based on the Poison or NBD (negative-binomial) distribution, they originated from research on stochastic interpurchase times and proved to be very accurate in fitting of the aggregated data describing frequently purchased goods (Dunn et al., 1983; Wagner and Taudes, 1987; Gupta, 1991; Agrawal and Smith, 1996, Grange 1998). However, their basic assumption on exponential (or gamma-exponential) distribution of the inter-arrival times does not allow to take into account quasi-periodicity in purchases/visits, which becomes a vital issue for personalization of marketing decisions. To overcome this problem, Telang et al. (2004) proposed recently a hierarchical probabilistic model of user's repeat visits that incorporates Weibull, Laplace and doubleexponential distribution mixture to account for users' schedule. This model was successfully applied to forecasting of visits to massively popular Internet web sites, but it seems to be hardly applied to intermittent demand modelling, which possesses the above mentioned specificities.

In this paper, the problem of quasi-periodicity is solved within Bayesian framework, using the generalized beta-binomial demand distribution (GBBD) developed in our previous paper (Dolgui et al., 2004). The demand repeatability is described by a historical data array corresponding to a typical time period, which also allows generating personalised forecasts using non-aggregated demand data.

\section{QUASI- PERIODIC DEMAND MODEL}

Let us assume that the intermittent demand patterns posses natural regularity over the time period $T$ ( 24 hours, a week, etc.), the examined time interval $[0, k T]$ includes $k$ periods, and the period $T$ is uniformly divided in $m$ time segments (1 hour, a day, etc.). Following our previous paper (Dolgui et al., 2004), let us also assume that, within these segments, the demand data are arranged in an integer matrix $\left\{s_{i j}, i=1 \ldots k, i=1 \ldots m\right\}$, where each observed value $s_{i j}$ is presented as a sum of $n$ binary random variables $s_{i j}=\sum_{l=1}^{n} b_{i j l}$ obtained via the Bernoulli trials with the random probabilities $p_{j}$ following the generalized beta distribution
$f(p)=\frac{1}{B\left(\alpha_{j}, \beta_{j}\right)\left(\pi_{1}-\pi_{0}\right)} \cdot\left(\frac{p-\pi_{0}}{\pi_{1}-\pi_{0}}\right)^{\alpha_{j}-1}\left(\frac{\pi_{1}-p}{\pi_{1}-\pi_{0}}\right)^{\beta_{j}-1}$

where $B(\alpha, \beta)$ is the complete beta function; $\alpha, \beta$ and $\pi_{0}, \pi_{1}$ are the shape and range distribution parameters respectively. It should be noted that the above formulation accounts the quasi-periodicity via the parameter arrays $\left\{\alpha_{j}, \beta_{j}, j=1: m\right\}$ defined and varying on the time period $[0, T]$, however below we also apply the Bayesian framework based on the historical data averaged for the similar time periods.

Under such assumptions, the columns of the demand matrix $\left\{s_{i j}\right\}$ obey the generalized beta-binomial demand distribution, which is computed using the above proposition, where $P_{r}^{(j)}$ is the probability that the demand for the $j$-th segment is equal to $r$.

Proposition 1. For the general-beta prior, the probability distribution of the intermittent demand can be represented as the weighted sum of the shifted betabinomial pdfs

$$
P_{r}^{(j)}=\sum_{l=0}^{n} w_{r l} \cdot\left(\begin{array}{l}
n \\
l
\end{array}\right) \frac{B\left(\alpha_{j}+l, \beta_{j}+n-l\right)}{B\left(\alpha_{j}, \beta_{j}\right)}
$$

where $P_{r}^{(j)} \in\left[\pi_{0}, \pi_{1}\right]$ is the probability that the demand value for the $j$-th segment of the period is equal to $r$, and the weights are computed as

$w_{r l}=\sum_{s=\max (r, l)}^{\min (n, r+l)}\left(\begin{array}{c}l \\ s-r\end{array}\right) \cdot\left(\begin{array}{c}n-l \\ s-l\end{array}\right) \cdot \pi_{0}^{s-l}\left(1-\pi_{0}\right)^{n-s} \pi_{1}^{l+r}\left(1-\pi_{1}\right)^{s-r}$

Proof of Proposition 1 is based on application of the hypergeometric expansion and properties of the complete beta function. First, using the linear transformation $p=\pi_{0}+\Delta \cdot \theta$ with the standard-beta random variable $\theta \in[0,1]$ and the width parameter $\Delta=\pi_{1}-\pi_{0}$, the expression for the marginal distribution of $r$ is rewritten as

$$
P_{r}=\frac{1}{B(\alpha, \beta)} \cdot\left(\begin{array}{l}
n \\
r
\end{array}\right) \cdot \int_{0}^{1}\left(\pi_{0}+\Delta \cdot \theta\right)^{r}\left(\rho_{0}-\Delta \cdot \theta\right)^{n-r} \theta^{\alpha-1}(1-\theta)^{\beta-1} d \theta
$$

where $\rho_{0}=1-\pi_{0}$ and the subscripts " $j$ " are omitted for brevity. Then, the product $\left(\pi_{0}+\Delta \cdot \theta\right)^{r}\left(\rho_{0}+\Delta \cdot \theta\right)^{n-r}$ is expanded into the sum $\sum_{l=0}^{n} w_{r l} \theta^{l}(1-\theta)^{n-l}$, where the coefficients $w_{r l}$ are using the Binomial theorem. Finally, to derive Eq.(2), the obtained sum of the integrals is expressed via the beta-functions. For computing convenience, the ratios of the betafunctions $B\left(\alpha_{j}+l, \beta_{j}+n-l\right) / B\left(\alpha_{j}, \beta_{j}\right)$ can be further simplified and Eq.(2) can be rewritten as 


$$
P_{r}^{(j)}=\sum_{l=1}^{n} w_{r l} \cdot\left(\begin{array}{l}
n \\
l
\end{array}\right) \frac{\prod_{s=0}^{l-1}\left(\alpha_{j}+s\right) \prod_{s=0}^{l-1}\left(\beta_{j}+n-s\right)}{\prod_{s=0}^{n-1}\left(\alpha_{j}+\beta_{j}+s\right)}
$$

Using the same approach, the demand mean $E(r)$ can be expressed via the conditional expectation as $E_{\theta}[E(r \mid \theta)]=n \pi_{0}+\Delta \cdot E(n \theta)$, that after substitution of $E(\theta)$, yields the following average demand value

$$
E(r)=\pi_{0} \cdot n \beta_{j} /\left(\alpha_{j}+\beta_{j}\right)+\pi_{1} \cdot n \alpha_{j} /\left(\alpha_{j}+\beta_{j}\right) .
$$

where $n \alpha /(\alpha+\beta)$ and $n \beta /(\alpha+\beta)$ can be interpreted as the average success/failure number for the standard BBD-model. Similarly, the demand variance $V(r)$ be expressed via the conditional mean and variance as $V(r)=V_{\theta}(n p \mid \theta)+E_{\theta}(n p(1-p) \mid \theta)$ that after computing of the conditional components and substitution of $E(\theta)$ and $V(\theta)$ yields

$$
V(r)=V_{0}+\pi_{0}\left(1-\pi_{0}\right) \cdot \frac{n \beta_{j}}{\alpha_{j}+\beta_{j}}+\pi_{1}\left(1-\pi_{1}\right) \cdot \frac{n \alpha_{j}}{\alpha_{j}+\beta_{j}},
$$

where the first term

$$
V_{0}=\Delta^{2} \cdot n \alpha_{j} \beta_{j}\left(\alpha_{j}+\beta_{j}+n_{j}\right) /\left(\alpha_{j}+\beta_{j}\right)^{2}\left(\alpha_{j}+\beta_{j}+1\right)
$$

can be treated as the weighted variance of the standard beta-binomial distribution with the scale factor equal to the square range of $p$, and the remainder ones are the weighted variances of the binomial distribution with parameters $\pi_{0}, \pi_{1}$.

\section{ESTIMATION OF MODEL PARAMETERS}

The proposed demand model includes two types of parameters, the shape parameters $\alpha_{j}, \beta_{j}$ varying over the period $T$ and the range parameters $\pi_{0}, \pi_{1}$, which are assumed to be similar for all the time segments. To estimate them, let us apply the MM technique combined with the minimisation of the Pirson's $\chi^{2}$ statistics, sequentially considering cases of known and unknown $\pi_{0}, \pi_{1}$ (such approach simplifies the general identification procedure, which adjusts the range parameters in the outer loop while the inner loop tunes the shape parameters).

If the probability range $\left[\pi_{0}, \pi_{1}\right]$ is assumed to be known, the remaining model parameters $\alpha, \beta$ should ensure equality of the mean $\pi^{*}$ and variance $\delta^{*}$ for the model and normalised demand data $\left\{s_{i j} / n\right\}$, i.e. $\pi^{*}=E(r) / n ; \delta^{*}=V(r) / n$, where the explicit expressions for $E(r)$ and $V(r)$ are given in Section 2 and the subscript " $j$ " is omitted. Then, from the equation for the first moment, the fractions $\alpha /(\alpha+\beta)$ and $\beta /(\alpha+\beta)$ may be expressed respectively as $\left(\pi^{*}-\pi_{0}\right) / \Delta$ and $\left(\pi_{1}-\pi^{*}\right) / \Delta$. Then, after substitu- tion, the equation for the second moment may be solved for $(\alpha+\beta)$. So, the parameters of interest are computed as

$$
\alpha=\eta \cdot\left(\pi^{*}-\pi_{0}\right) / \Delta ; \quad \beta=\eta \cdot\left(\pi_{1}-\pi^{*}\right) / \Delta
$$

where

$$
\eta=(n-1) \frac{\delta^{*}-\pi^{*}\left(1-\pi^{*}\right)}{\left(\pi^{*}-\pi_{0}\right)\left(\pi_{1}-\pi^{*}\right)}-1
$$

Then, let us release the assumption concerning the known probability range and estimate the parameters $\pi_{0}, \pi_{1}$ minimizing the Pirson's $\chi^{2}$-statistics that describes goodness-of-fit for the empirical distribution $\left\{p_{r}^{*}\right\}$. The corresponding optimisation problem may be written as

$$
F=\sum_{r=0}^{n}\left(p_{r}^{*}\right)^{2} / P_{r}\left(\alpha, \beta, \pi_{0}, \pi_{1}\right) \rightarrow \min _{\alpha, \beta, \pi_{0}, \pi_{1}}
$$

subject to

$$
m\left(\alpha, \beta, p_{0}, p_{1}\right)=n p^{*} ; d\left(\alpha, \beta, p_{0}, p_{1}\right)=n \delta^{*} .
$$

where $\left\{p_{k}^{*}, k=\overline{1, n}\right\}$ are the empirical frequencies and $m(),. d($.$) denote respectively the mean and dis-$ persion of the considered probability distribution model. It should be noted that the objective function $F\left(\right.$.) is directly related to the standard $\chi^{2}$-statistics. Since the desired parameters $\pi_{0}, \pi_{1}$ must belong to the intervals $\left[0, \pi^{*}\right)$ and $\left(\pi^{*}, 1\right]$, this optimisation problem has been solved numerically, by sequentially applying a one-dimensional search for $\pi_{0}, \pi_{1}$ and re-computing $\alpha, \beta$ from the expressions (6).

As follows from our research, the algorithms is rather sensitive to initial estimates. Thus, to ensure good convergence, the developed numerical routines includes the extremum localization step (using gridbased search) and exponential smoothing of the updates for the range parameters.

\section{FORECASTING DEMAND DISTRIBUTION}

Let assume now that, in addition to the parameter arrays $\left\{\alpha_{j}, \beta_{j}, j=1: m\right\}$, the quasi-periodicity is also described by the historical array $\left\{v_{j}, j=1: m\right\}$ obtained by the demand averaging on the similar time segments. Then it can be proved that the Bayesian approach leads to the following posterior distribution for the probabilities of the non-zero demand binary components $b_{j l}$

$$
f\left(p \mid v_{j}\right)=\frac{p^{v_{j}}\left(p-\pi_{0}\right)^{\alpha_{j}-1}(1-p)^{n-v_{j}}\left(\pi_{1}-p\right)^{\beta_{j}-1}}{\int_{\pi_{0}}^{\pi_{1}} p^{v_{j}}\left(p-\pi_{0}\right)^{\alpha_{j}-1}(1-p)^{n-v_{j}}\left(\pi_{1}-p\right)^{\beta_{j}-1} d p}
$$

that is computed using the following proposition. 
Proposition 2. For the adopted demand model, the Bayesian forecasting distribution can be represented as the weighted sum of the shifted beta pdfs

$$
f(p \mid v)=\sum_{l=0}^{n} \omega_{v l} \cdot \frac{p^{\alpha_{j}+l-1} \cdot(1-p)^{\beta_{j}+n-l-1}}{B\left(\alpha_{j}+l, \beta_{j}+n-l\right)}
$$

and the mean square optimal predictor function is

$$
\hat{p}(v)=\sum_{l=0}^{n} \omega_{v l} \cdot\left(\alpha_{j}+l\right) /\left(\alpha_{j}+\beta_{j}+n\right),
$$

where the weights are expressed as

$$
\omega_{v l}=\frac{\left(\begin{array}{l}
n \\
l
\end{array}\right) w_{v i} B(\alpha+l, \beta+n-l)}{\sum_{j=0}^{n}\left(\begin{array}{l}
n \\
j
\end{array}\right) w_{v j} B(\alpha+j, \beta+n-j)} .
$$

Proof of Proposition 2 uses the same technique as Proposition 1 (variable expression via $\theta$, hypergeometric expansion, and relevant transformation). It has been also proved that the developed predictor ensures the following mean square forecast error

$$
\begin{aligned}
& \varepsilon^{2}(p)=\frac{\alpha^{[2+]}}{(\alpha+\beta)^{[2+]}-} \\
& \sum_{v=0}^{n}\left(\left(\sum_{l=0}^{n} \omega_{v l} \frac{\alpha+l}{\alpha+\beta+n}\right)^{2} \cdot \sum_{j=0}^{n} w_{v l}\left(\begin{array}{l}
n \\
l
\end{array}\right) \frac{\alpha^{[l+]} \beta^{[(n-l)+]}}{(\alpha+\beta)^{[n+]}}\right)
\end{aligned}
$$

where $\alpha^{[l+]}$ denotes the ascending factorial defined as the product $\alpha(\alpha+1) \cdot \ldots \cdot(\alpha+l-1)$.

\section{SIMULATION STUDY}

To demonstrate the applicability of the developed model to describing the demand quasi-periodicity, a simulation study was performed. Following research of Telang et al. (2004), it was considered a demand model with the time period 24 hours and hourly data aggregation, with the smallest time unit within the segment of 3 minutes that corresponds to a single binary demand fraction, i.e. $m=24$ and $n=20$. It was assumed that the prior probability of the non-zero demand within each time unit follows the beta distribution with "flat" parameters $\alpha=0.02, \beta=4.98$, while the repeatability is described by the historical

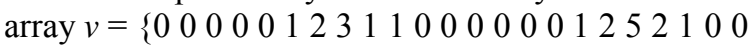
$0\}$ with two evident peaks during the 24-hour period. It should be stressed that both the prior model and the historical data yield the same average demand value, 0.08 units/hour, which is low enough to describe orders of the "slow-moving" items considered in this paper.

Simulation results are presented in Figs. 1, 2 and Table 1. As expected, the demand patterns (Fig. 1) exhibit the quasi-periodicity having strong concentration of the non-zero values close to the historical data peaks; at the same time, they posses typical intermittent properties (large proportion of zeros and great variability of the non-zeros, see Table 1). Also, the corresponding inter-arrival-time distribution (Fig. 2) possesses a bi-modal shape, which can not be properly described by the exponential function adopted in the classical negative-binomial model (Agrawal and Smith, 1996; Telang et al. 2004).

Hence, the simulation study confirms appropriateness of the proposed approach in modelling of the quasiperiodic demand patterns. Besides, the proposed model incorporates less statistical hierarchy levels in comparison with the known one (Telang et al., 2004) and relies on robust identification routines, which can compensate the reporting errors (Dolgui et al., 2004).

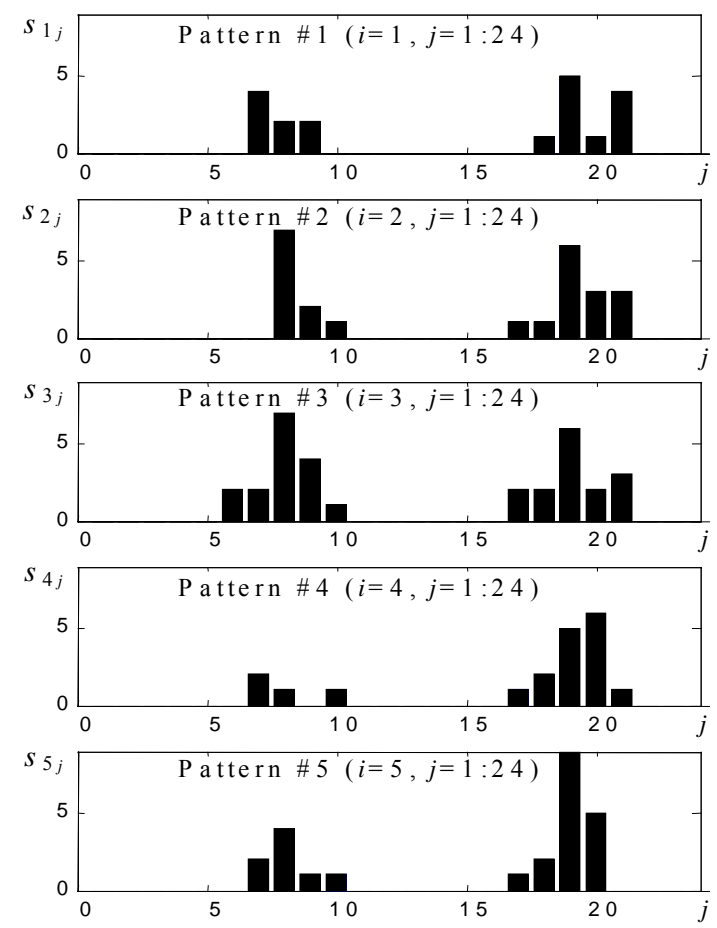

Fig. 1. Example patterns of quasi-periodic demand

Table 1. Distribution of the demand values

\begin{tabular}{c|ccccccc}
\hline$r$ & $P_{0}$ & $P_{1}$ & $P_{2}$ & $P_{3}$ & $P_{4}$ & $P_{5}$ & $P_{6+}$ \\
\hline$P_{r}$ & 0.967 & 0.016 & 0.007 & 0.004 & 0.002 & 0.001 & 0.004 \\
\hline
\end{tabular}

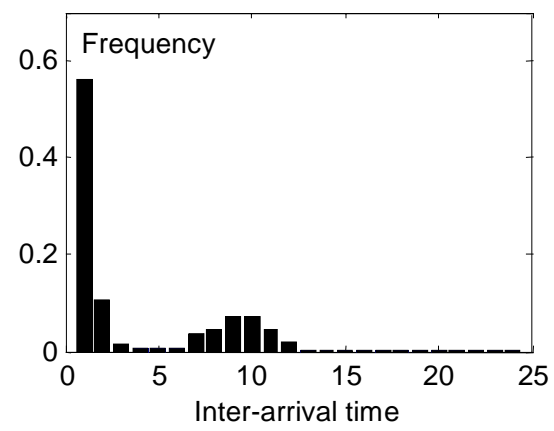

Fig. 2. Distribution of the time intervals 
Table 2. Demand data for three time periods

\begin{tabular}{c|cccccccccccc}
\hline Segment & 1 & 2 & 3 & 4 & 5 & 6 & 7 & 8 & 9 & 10 & 11 & 12 \\
\hline Period \#1 & 3 & 0 & 2 & 0 & 0 & 0 & 0 & 1 & 0 & 0 & 1 & 2 \\
Period \#2 & 0 & 1 & 0 & 0 & 1 & 1 & 2 & 1 & 0 & 2 & 0 & 0 \\
Period \#3 & 0 & 1 & 1 & 2 & 2 & 2 & 1 & 0 & 0 & 2 & 0 & 0 \\
\hline
\end{tabular}

Table 3. Fitting of the demand data

\begin{tabular}{|c|c|c|c|c|c|}
\hline \multicolumn{2}{|c|}{ Data } & \multicolumn{4}{|c|}{ Model distributions } \\
\hline$r$ & $p_{r}{ }^{*}$ & $n=3$ & $n=4$ & $n=5$ & $n=6$ \\
\hline 0 & 0.541 & 0.533 & 0.523 & 0.518 & 0.516 \\
\hline 1 & 0.250 & 0.272 & 0.301 & 0.310 & 0.315 \\
\hline 2 & 0.167 & 0.142 & 0.130 & 0.126 & 0.124 \\
\hline 3 & 0.042 & 0.050 & 0.040 & 0.037 & 0.036 \\
\hline & $\alpha$ & 0.729 & 1.263 & 1.701 & 2.068 \\
\hline & $\beta$ & 2.050 & 5.157 & 9.108 & 13.70 \\
\hline & $\ell$ & 0.154 & 0.510 & 0.663 & 0.741 \\
\hline
\end{tabular}

Table 4. Forecast based on the previous period

\begin{tabular}{c|ccccc}
\hline \multirow{2}{*}{ Segment } & \multicolumn{4}{|c}{ Forecast } \\
\cline { 2 - 5 } & $n=3$ & $n=4$ & $n=5$ & $n=6$ \\
\hline 1 & 0.408 & 0.510 & 0.559 & 0.589 \\
2 & 0.957 & 0.906 & 0.883 & 0.870 \\
3 & 0.408 & 0.510 & 0.559 & 0.589 \\
4 & 0.408 & 0.510 & 0.559 & 0.589 \\
5 & 0.957 & 0.906 & 0.883 & 0.870 \\
6 & 0.957 & 0.906 & 0.883 & 0.870 \\
7 & 1.478 & 1.293 & 1.202 & 1.148 \\
8 & 0.958 & 0.906 & 0.883 & 0.870 \\
9 & 0.408 & 0.510 & 0.559 & 0.589 \\
10 & 1.478 & 1.292 & 1.202 & 1.148 \\
11 & 0.408 & 0.510 & 0.559 & 0.589 \\
12 & 0.408 & 0.510 & 0.559 & 0.589 \\
\hline$\varepsilon$ & 0.791 & 0.801 & 0.812 & 0.819 \\
\hline
\end{tabular}

Table 5. Forecast based on the weighted sum of the previous period and previous segment

\begin{tabular}{c|ccccc}
\hline \multirow{2}{*}{ Segment } & \multicolumn{4}{|c}{ Forecast } \\
\cline { 2 - 5 } & $n=3$ & $n=4$ & $n=5$ & $n=6$ \\
\hline 1 & 0.408 & 0.509 & 0.559 & 0.5887 \\
2 & 1.340 & 1.268 & 1.236 & 1.2177 \\
3 & 1.531 & 1.449 & 1.412 & 1.3916 \\
4 & 1.531 & 1.449 & 1.412 & 1.3916 \\
5 & 2.364 & 2.068 & 1.923 & 1.8370 \\
6 & 2.364 & 2.068 & 1.923 & 1.8370 \\
7 & 1.478 & 1.292 & 1.202 & 1.1481 \\
8 & 0.957 & 0.906 & 0.883 & 0.8698 \\
9 & 0.408 & 0.509 & 0.559 & 0.5887 \\
10 & 2.660 & 2.326 & 2.1634 & 2.0666 \\
11 & 1.149 & 1.087 & 1.059 & 1.0437 \\
12 & 0.408 & 0.509 & 0.559 & 0.5887 \\
\hline$\varepsilon$ & 0.390 & 0.509 & 0.450 & 0.474 \\
\hline
\end{tabular}
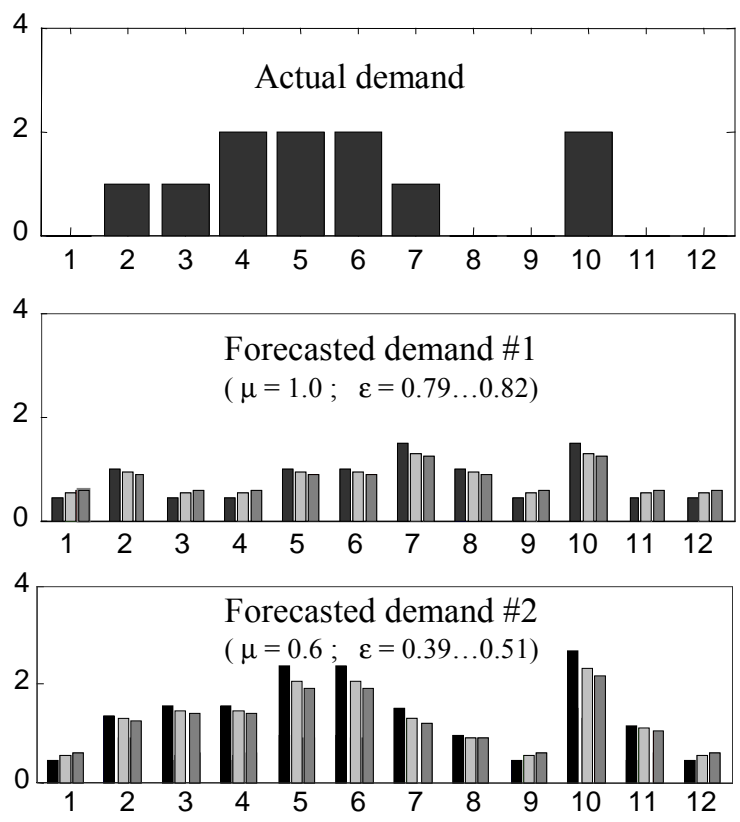

Fig. 3. Actual demand versus forecasts for different values of $n$ and $\mu(-n=3 ; \square-n=4 ;-n=5)$

\section{APPLICATION EXAMPLE}

To validate the efficiency of the proposed technique, there were explored a data set for a car spare part sold in Australia (Snyder, 2002), which demonstrates typical intermittent properties and possesses an obvious quasi-periodicity within the 12-months periods (Table 2). The aggregation time-segment was assumed to be equal to one month, and the demand values for 36 months were available. For comparison purposes, there were generated two forecasts, which differ in methods of constructing the historical arrays employed in the Bayesian expressions.

The demand model parameters $\left\{\alpha, \beta, \pi_{0}, \pi_{1}, n\right\}$ incorporated in the prior distribution (1) were estimated using the numerical routines described in Section 3, using the statistical data for the months 1-24. There were investigated several cases that differ by parameter $n$ (number of binary items in the demand representation). The minimum value of $n$ was set as the upper demand level for these segments. The estimated results are presented in Table 3 , which confirms that such selection of $n$ ensures the best fitting of the experimental data, but for the practical reasons this value should be slightly increased to allow some larger demands, that were not observed in a particular experimental data set.

Using the demand statistical model extracted from the months 1-24, there were generated two Bayesian forecasts for the months 25-36. For the first forecast (Table 4), the historical array was created by straightforward coping the demands for the months 13-24, which is the most natural way of accounting the sea- 
sonal repeatability (since the demand of the month 25 should be in certain degree similar to the demand of the month 13, etc.). Such approach yielded the forecast error about 0.8 items/months, which slightly increases while increasing $n$.

The second forecast (Table 5) relies on another historical array, which is created from both the previous-year and the previous-month demand values (with certain weights $\mu$ and $1-\mu$ ). The reason behind this is that, in addition to the seasonality, it should be also strong correlation between the demands of the successive months. As follows from our research, for this particular example, the best forecast is obtained for $\mu \approx 0.6$, which corresponds to the forecast error $0.39 \ldots 0.47$ items $/$ month. It is almost twice lower, than in the previous case.

Hence, this application example confirms the applicability of the developed method to the modelling of real-life intermittent demand patterns and efficiency of the relevant Bayesian forecast.

\section{CONCLUSION}

Forecasting the intermittent demand for service parts and high-priced capital goods is a challenging problem of the inventory management. Current practice in such demand modelling favours the exponential smoothing of the demand values or applying exponential smoothing separately to the intervals between nonzero demands and their sizes (Croston's method). An alternative recommended in inventory control literature is based on the stochastic demand modelling using the negative binominal distribution that assumes exponentially distributed inter-arrival times.

In this paper, it is proposed a new stochastic model, which describes quasi-periodic intermittent demand patterns with essentially non-exponential (polymodal) distribution of the inter-arrival times. It is based on generalized beta-binomial distribution and the Bayesian inference using the historical data array describing the demand repeatability within the time periods. For this model, there were derived explicit expressions for the forecast distributions, its moments and relevant Bayesian risk. The efficiency of the proposed approach is confirmed by computer simulation and is illustrated by an application example for modelling of the demand patterns for car spare parts.

\section{REFERENCES}

Agrawal, N., and S.A. Smith, (1996). Estimating Negative Binomial Demand for Retail Inventory Management with Unobservable Lost Sales, $\mathrm{Na}$ val Research Logistics, Vol. 43, pp. 839-861.
Croston, J. D. (1972). Forecasting and stock control for intermittent demands. Operational Research Quarterly, 23 (3), 289- 303.

Dunn R., S. Reader, and N. Wrigley (1983), An investigation of the assumptions of the NBD model as applied to purchasing at individual stores, Applied Statistics, 32, 249-59.

Dolgui, A., A. Pashkevich and M. Pashkevich (2004). Modeling Demand for Inventory Management of Slow-Moving Items in Case of Reporting Errors. Proceedings of 11th IFAC Symposium on Information Control Problems in Manufacturing, Salvador, Brazil, April 5-7, 2004, 6pp.

Grange, F (1998). Challenges in modeling demand for inventory optimization of slow-moving items. Proceedings of the IEEE Winter Simulation Conference, pp. 1211-1217.

Gupta S. (1991), Stochastic models of interpurchase time with time dependent covariates, Journal of Marketing Research, 28 (February), 1-15.

Harris, T. (1997). Optimized Inventory Management, Production and Inventory Management Journal, Vol. 38(1), pp. 22-25.

Johnston, F. R. and Boylan, J. E. (1996). Forecasting for items with intermittent demand. Journal of the Operational Research Society, 47, 113-121.

Shenstone, L. and R.J. Hyndman (2003). Stochastic models underlying Croston's method for intermittent demand forecasting. Working paper 1/2003, Department of Econometrics and Business Statistics, Monash University, Australia (http://ideas.repec.org/p/msh/ebswps /20031.html)

Silver, E.A., D.F. Pykeand and R. Peterson (1998). Inventory management and production planning and scheduling, Wiley, New York.

Snyder, S. (2002). Forecasting Sales of Slow and Fast Moving Inventories, European Journal of Operational Research, Vol. 140, pp. 684-699.

Syntetos, A. A. and Boylan, J. E. (2001), On the bias of intermittent demand estimates, International Journal of Production Economics Vol. 71(1-3), pp. 457-466.

Telang, R., P. Boatwright, and T. Mukhopadhyay (2004). A Mixture Model for Internet Search Engine Visits, Journal of Marketing Research, Vol. 41(2), pp. 206-214.

Wagner, U., and A. Taudes (1987). Stochastic Models of Consumer Behaviour, European Journal of Operational Research, Vol, 29(1), pp. 1-23.

Willemain, T.R., C.N. Smart, J.H. Shockor, and P.A. DeSautels (1994). Forecasting intermittent demand in manufacturing: A comparative evaluation of Croston's method. International Journal of Forecasting Vol. 10(4), pp. 529-538.

Willemain T.R., C.N. Smart and H.F. Schwarz (2004). A new approach to forecasting intermittent demand for service parts inventories, International Journal of Forecasting, Vol. 20(3), pp. 375-387 